\title{
Mathematical and Dynamic Analysis of a Prey-Predator Model in the Presence of Alternative Prey with Impulsive State Feedback Control
}

\author{
Chuanjun Dai ${ }^{1}$ and Min Zhao ${ }^{2}$ \\ ${ }^{1}$ School of Mathematics and Information Science, Wenzhou University, Zhejiang, Wenzhou 325035, China \\ ${ }^{2}$ School of Life and Environmental Science, Wenzhou University, Zhejiang, Wenzhou 325035, China
}

Correspondence should be addressed to Min Zhao, zmcn@tom.com

Received 5 October 2011; Revised 21 December 2011; Accepted 21 February 2012

Academic Editor: Xue He

Copyright @ 92012 C. Dai and M. Zhao. This is an open access article distributed under the Creative Commons Attribution License, which permits unrestricted use, distribution, and reproduction in any medium, provided the original work is properly cited.

The dynamic complexities of a prey-predator system in the presence of alternative prey with impulsive state feedback control are studied analytically and numerically. By using the analogue of the Poincaré criterion, sufficient conditions for the existence and stability of semitrivial periodic solutions can be obtained. Furthermore, the corresponding bifurcation diagrams and phase diagrams are investigated by means of numerical simulations which illustrate the feasibility of the main results.

\section{Introduction}

Since the pioneering work of Lotka and Volterra, the theoretical investigation of predatorprey systems in mathematical ecology has advanced greatly. The theory of impulsive differential equations is one of the theoretical components of this investigation. The study of impulsive differential equations mainly concerns the properties of their solutions, such as existence, uniqueness, stability, boundedness, and periodicity [1].

In fact, in the natural world, there is sometimes a need to control a population to a reasonable level because otherwise this population might lead other populations to decrease or even to become extinct [2]. These processes may need to be modeled using impulsive systems rather than continuous systems because of abrupt jumps that occur during their evolution. Generally speaking, there are three kinds of systems with impulsive perturbations: systems with impulses at fixed times, systems with impulses at variable times, and autonomous impulsive systems [3]. In recent years, most investigations of impulsive differential equations have concentrated on systems with impulses at fixed times [4-12], 
while the other two kinds of impulsive differential equations have been relatively less studied. However, in many practical cases, impulses often occur at state-dependent rather than at fixed times. For example, it may be desired to control a population size by catching or crop-dusting and releasing a predator when prey numbers reach a threshold value.

As is well known, significant theoretical development has recently been achieved in the bifurcation theory of continuous dynamic systems [13-18]. This paper will also consider the bifurcation behavior of systems with impulses. Recently, Lakmeche and Arino [19] transformed the problem of finding a periodic solution into a fixed-point problem and discussed the bifurcation of periodic solutions from trivial solutions, as well as obtaining the existence conditions for a positive period-1 solution. Tang and Chen [20] obtained a completed expression for a period-1 solution and discussed the bifurcation of period solutions numerically using a discrete dynamic system determined by a stroboscopic map. Many papers have been devoted to the analysis of mathematical models with state-dependent impulsive effects. For instance, Tang and others have studied the dynamic behaviors of predator-prey systems with impulsive-state feedback control and have determined the existence and stability of positive periodic solutions using the Poincare map and the properties of the Lambert $W$ function [21-26].

Through a long course of investigation, many authors have emphasized that the presence of alternative foods can effect biological control through a variety of mechanisms. For example, the presence of one prey population can have negative effects on another prey population by enabling the population of a shared predator to increase, thus leading to higher predation rates upon both prey items [27]. In contrast, the alternative prey can have a positive effect on population densities of the focal prey, in that the alternative prey can reduce predation on the focal prey because of predator preference for alternative prey resources [27].

This research uses the method of impulsive perturbations and the presence of alternative prey to investigate the following predator-prey model with state-dependent impulsive effects:

$$
\begin{gathered}
\frac{d N}{d T}=r N\left(1-\frac{N}{k}\right)-\frac{\alpha_{1} N P}{a+N}, \quad \frac{d P}{d T}=\frac{\beta_{1} \alpha_{1} N P}{a+N}+d_{1} P\left(1-\frac{N}{k}\right)-\gamma_{1} P, \quad N \neq H \\
\Delta N=-c N, \quad \Delta P=e P+f, \quad N=H,
\end{gathered}
$$

where $N(T), P(T)$ represent the densities or biomasses of the prey and the predator, respectively at time $T \cdot r N(1-N / K)$ is the logistic equation, which is often used to describe the prey population increment in the absence of predator. The logistic equation has been applied in a wide range of ecological system situations, especially predator-prey system, and its theoretical assumptions permit population growth to be halted by a resource limitation, where $K$ is the environmental carrying capacity of the prey allowed by the limiting resource and $r$ denotes the intrinsic growth rate of the prey. $\alpha_{1} N P /(a+N)$ is the Holling type II functional response, which is used to describe the average feeding rate of a predator when the predator spends some time searching for prey and some time, exclusive of searching, processing each captured prey item, where $\alpha_{1}$ is the predation coefficient and $a$ is the halfsaturation constant. Furthermore, it should be noted that the feeding rate is unaffected by predator abundance, but the Holling type II functional response has been used and has stood as the null model upon which predator-prey theory is based. $d_{1} P(1-N / K)$ denotes that the part of the predator population increments come from the alternative prey, where $d_{1}$ is the digestion factor relative to the alternative prey. For simplicity, it is compulsory to assume that 
the alternative prey has a high abundance so that the predator can feed it, but the feeding rate of the alternative prey is affected by the prey $N$, that is to say when the value of $N$ tends to the environmental carrying capacity $K$, the mass of the alternative prey consumed will tend to zero. $\gamma_{1}$ is the mortality rate of the predator, respectively. $\beta_{1}$ is a conversion factor, and it is assumed that $\beta_{1}<1$ because the whole biomass of the prey is not converted into the biomass of the predator.

In practice, we can control the population size by catching (poisoning) the prey (i.e., the pest) and releasing the predator (i.e., the natural enemy) when the amount of the prey reaches a threshold [28]. Because this situation does not occur at a fixed time, the usual kind of fixed-time control strategy may not be effective. Therefore, a state-based feedback control strategy is introduced in our paper. When the numbers of prey $N$ reach a threshold value $H$, the decision maker of biological treatment technology must implement impulsive control strategy that can better handle the relationship between the prey $N$ and the predator $P$. Hence, we not only harvest a certain number of the prey $N$, but also release a certain amount of $P$ by the use of indirect or direct way, where $1>c>0, e>0, f \geq 0$.

It is convenient at the outset to rescale the system described above by introducing $N=a x, P=r a y / \alpha_{1}, T=t / r$; then system (1.1) becomes:

$$
\begin{gathered}
\frac{d x}{d t}=x\left(1-\frac{a x}{K}\right)-\frac{x y}{1+x}, \quad \frac{d y}{d t}=\frac{\beta_{1} \alpha_{1} x y}{r(1+x)}+\left(\frac{d_{1}}{r}\right) y\left(1-\frac{a x}{K}\right)-\left(\frac{\gamma_{1}}{r}\right) y, \quad a x \neq H \\
\Delta x=-c x, \quad \Delta y=e y+\frac{\alpha_{1} f}{r a}, \quad a x=H .
\end{gathered}
$$

Letting $K / a=k, \beta_{1} \alpha_{1} / r=b, d_{1} / r=d, \gamma_{1} / r=m, c=p, e=q, \alpha_{1} f / r a=\tau, H / a=h$, then system (1.2) can be written in the following form:

$$
\begin{gathered}
\frac{d x}{d t}=x\left(1-\frac{x}{k}\right)-\frac{x y}{1+x}, \quad \frac{d y}{d t}=\frac{b x y}{1+x}+d y\left(1-\frac{x}{k}\right)-m y, \quad x \neq h \\
\Delta x=-p x, \quad \Delta y=q y+\tau, \quad x=h .
\end{gathered}
$$

The rest of this paper is organized as follows. In Section 2, some lemmas that are frequently used in the following discussions are presented, and the existence and stability of a positive periodic solution of system (1.3) are stated and proved. In Section 3, the results of numerical analysis are reported to illustrate the theoretical results. Finally, conclusions and remarks are provided.

\section{Analysis of the System}

To discuss the dynamic behavior of system (1.3), a Poincaré map must be constructed. First, consider the vector field of system (1.3). Let $S_{0}=\{(x, y) \mid x=(1-p) h, y \geq 0\}$ and $S_{1}=$ $\{(x, y) \mid x=h, y \geq 0\}$. Then the horizontal isocline $y=(1-x / k)(1+x)$ intersects sections $S_{0}$ and $S_{1}$ at $B(h,(1-h / k)(1-h))$ and $A((1-p) h,(1-(1-p) h / k)(1+(1-p) h))$, respectively. The segment $A B$ is represented by $y=(1-x / k)(1+x)$, where $(1-p) h<x<h$, and sections $S_{0}$ and $S_{1}$ intersect $y=0$ at $C((1-p) h, 0)$ and $D(h, 0)$, respectively. It follows that $\Omega_{1}=\Omega \cup C D$ and $\Omega=\{(x, y) \mid 0<y<(1-x / k)(1+x),(1-p) h<x<h\}$. It is easy to see that $d x=0, d y<0$ are satisfied at a point $(x, y) \in A B$. On the one hand, any orbit passing through segment 
$A B$ enters $\Omega$ as $t$ increases and then departs from $\Omega$ by passing through $B D$. On the other hand, any orbit beginning with the point $\left(x_{0}, 0\right) \in C D \subset \Omega_{1}$ keeps $y(t)=0$ and tends to $\infty$. However, for any point $(x, y) \in \Omega_{1}, d x / d t>0$, which is a major condition for the following discussion.

Next, choose sections $S_{0}$ and $S_{1}$ as Poincaré sections. First assume that the point $B_{k-1}^{+}\left((1-p) h, y_{k-1}^{+}\right)$is on section $S_{0}$. Because of the nature of the vector field of system (1.3), the trajectory with initial point $B_{k-1}^{+}$intersects section $S_{1}$ at point $B_{k}\left(h, y_{k}\right)$, where $y_{k}$ depends on $y_{k-1}^{+}$, letting $y_{k}=g\left(y_{k-1}^{+}\right)$. Then point $B_{k}$ jumps to point $B_{k}^{+}\left((1-p) h,(1+q) y_{k}+\tau\right)$ on $S_{0}$ on account of the impulsive effects. Hence, the following Poincaré map $P$ is obtained:

$$
y_{k}^{+}=(1+q) g\left(y_{k-1}^{+}\right)+\tau \text {. }
$$

Second, consider the other Poincare section. It is assumed that point $B_{k}\left(h, y_{k}\right)$ is on the Poincaré section $S_{1}$. Then $B_{K}^{+}\left((1-p) h,(1+q) y_{k}+\tau\right)$ is on section $S_{0}$ because of the impulsive influence, and the trajectory with initial point $B_{k}^{+}$intersects the Poincaré section $S_{1}$ at point $B_{k+1}\left(h, y_{k+1}\right)$, where $y_{k+1}^{+}$rests with $y_{k}$ and the parameters $q$ and $\tau$. Then another Poincaré map $P_{0}$ can be obtained as follows:

$$
y_{k+1}=g\left((1+q) y_{k}+\tau\right)=F\left(q, \tau, y_{k}\right) \text {. }
$$

To investigate the properties of system (1.3), the following lemma is introduced.

Lemma 2.1 (see [29]). The T-periodic solution $(x, y)=(\xi(t), \eta(t))$ of the system

$$
\begin{array}{rlrl}
\frac{d x}{d t} & =P(x, y), & \frac{d y}{d t}=Q(x, y), & \varphi(x, y) \neq 0, \\
\Delta x=\xi(x, y), & \Delta y=\eta(x, y), & \varphi(x, y)=0
\end{array}
$$

is orbitally asymptotically stable if the Floquet multiplier $\mu$ satisfies the condition $|\mu|<1$, where

$$
\mu=\prod_{k=1}^{n} \Delta_{k} \exp \left[\int_{0}^{T}\left(\frac{\partial P}{\partial x}(\xi(t), \eta(t))+\frac{\partial Q}{\partial y}(\xi(t), \eta(t))\right) d t\right]
$$

with

$$
\begin{aligned}
\Delta_{k}= & \frac{P_{+}((\partial \beta / \partial y)(\partial \phi / \partial x)-(\partial \beta / \partial x)(\partial \phi / \partial y)+(\partial \phi / \partial x))}{P(\partial \phi / \partial x)+Q(\partial \phi / \partial y)} \\
& +\frac{Q_{+}((\partial \alpha / \partial x)(\partial \phi / \partial y)-(\partial \alpha / \partial y)(\partial \phi / \partial x)+(\partial \phi / \partial y))}{P(\partial \phi / \partial x)+Q(\partial \phi / \partial y)}
\end{aligned}
$$

and $P, Q, \partial \alpha / \partial x, \partial \alpha / \partial y, \partial \beta / \partial x, \partial \beta / \partial y, \partial \phi / \partial x, \partial \phi / \partial y$ are calculated at points $\left(\xi\left(t_{k}\right), \eta\left(t_{k}\right)\right)$, $P_{+}=P\left(\xi\left(t_{k}^{+}\right), \eta\left(t_{k}^{+}\right)\right)$and $Q_{+}=Q\left(\xi\left(t_{k}^{+}\right), \eta\left(t_{k}^{+}\right)\right)$, where $\phi(x, y)$ is a sufficiently smooth function that grad $\phi(x, y) \neq 0$, and $t_{k}(k \in N)$ is the time of the $k$ th jump. 
Lemma 2.2 (see [30]). Let $F: R \times R \rightarrow R$ be a one-parameter family of the $C^{2}$ map satisfying

(i) $F(0, \mu)=0$,

(ii) $\partial F / \partial x(0,0)=1$,

(iii) $\partial^{2} F / \partial x \partial \mu(0,0)>0$,

(iv) $\partial^{2} F / \partial x^{2}(0,0)<0$.

Then $F$ has two branches of fixed points for $\mu$ near zero. The first branch is $x_{1}(\mu)=0$ for all $\mu$. The second bifurcating branch $x_{2}(\mu)$ changes its value from negative to positive as $\mu$ increases through $\mu=0$ with $x_{2}(0)=0$. The fixed points of the first branch are stable if $\mu<0$ and unstable if $\mu>0$, while those of the bifurcating branch have the opposite stability pattern.

Next, consider the case of system (1.3) without impulsive effect. From $x(1-x / k)-x y /(1+x)=$ 0 and $b x y /(1+x)+d y(1-x / k)-m y=0$, it can be determined that system (1.3) has two boundary equilibria $P_{0}(0,0), P_{1}(k, 0)$. Let $d+b-d / k-m>0, m-d>0$ and $(d+b-d / k-m)^{2}>(4 d / k)(m-d)$; then there are two positive interior equilibria $P_{1}^{*}\left(x_{1}^{*}, y_{1}^{*}\right), P_{2}^{*}\left(x_{2}^{*}, y_{2}^{*}\right)$, where

$$
\begin{gathered}
x_{1}^{*}=\left(\frac{k}{2 d}\right)\left[\left(d+b-\frac{d}{k}-m\right)+\sqrt{\left(d+b-\frac{d}{k}-m\right)^{2}-\left(\frac{4 d}{k}\right)(m-d)}\right], \\
y_{1}^{*}=\left(1-\frac{x_{1}^{*}}{k}\right)\left(1+x_{1}^{*}\right), \\
x_{2}^{*}=\left(\frac{k}{2 d}\right)\left[\left(d+b-\frac{d}{k}-m\right)-\sqrt{\left(d+b-\frac{d}{k}-m\right)^{2}-\left(\frac{4 d}{k}\right)(m-d)}\right], \\
y_{2}^{*}=\left(1-\frac{x_{2}^{*}}{k}\right)\left(1+x_{2}^{*}\right) .
\end{gathered}
$$

A direct calculation and stability analysis of these equilibrium points shows that $P_{1}^{*}\left(x_{1}^{*}, y_{1}^{*}\right)$ is a saddle, while $P_{2}^{*}\left(x_{2}^{*}, y_{2}^{*}\right)$ is an equilibrium point with index +1 , which is a stable positive focus when $\left(1+2 x_{2}^{*}\right) / k>1$. Throughout this paper, it is assumed that $d+b-d / k-m>0, m-d>0$ and $(d+b-d / k-m)^{2}>(4 d / k)(m-d)$ always hold on the basis of reasonable ecological practice. effect.

The following discussion refers to system (1.3), that is, the system with impulsive

\subsection{Case $\tau=0$}

In this subsection, some basic properties will be derived for the following subsystem of system (1.3), in which the predator $y(t)$ is absent:

$$
\begin{gathered}
\frac{d x}{d t}=x\left(1-\frac{x}{k}\right), \quad x \neq h, \\
\Delta x=-p x, \quad x=h .
\end{gathered}
$$

Setting $x_{0}=x(0)=(1-p) h$ leads to the following solution of system $(2.7): x(t)=k(1-$ $p) h \exp (t-n T) /(k-(1-p) h+(1-p) h \exp (t-n T))$. Let $T=\ln ((k-(1-p) h) /(k-h)(1-p))$; 
then $x(T)=h$ and $x\left(T^{+}\right)=(1-p) h$. This means that system (1.3) has the following semi-trivial periodic solution:

$$
\begin{gathered}
x(t)=\frac{k(1-p) h \exp (t-n T)}{k-(1-p) h+(1-p) h \exp (t-n T)}, \\
y(t)=0,
\end{gathered}
$$

where $t \in(n T,(n+1) T], n \in N$, which is implied by $(\xi(t), 0)$.

Next, the stability of this semi-trivial periodic solution will be investigated.

Theorem 2.3. The semi-trivial periodic solution (2.8) is said to be stable if

$$
0<q<(1-p)^{d-m}\left(\frac{k-(1-p) h}{k-h}\right)^{m-b k /(1+k)}\left(\frac{1+h}{1+(1-p) h}\right)^{-(b k /(1+k))}-1 .
$$

Proof. It is known that

$$
\begin{gathered}
P(x, y)=x\left(1-\frac{x}{k}\right)-\frac{x y}{(1+x)}, \quad Q(x, y)=\frac{b x y}{(1+x)}+d y\left(1-\frac{x}{k}\right)-m y, \\
\alpha(x, y)=-p x, \quad \beta(x, y)=q y, \quad \phi(x, y)=x-h, \\
(\xi(T), \eta(T))=(h, 0), \quad\left(\xi\left(T^{+}\right), \eta\left(T^{+}\right)\right)=((1-p) h, 0) .
\end{gathered}
$$

Then, using Lemma 2.1, by a straightforward calculation, it is possible to obtain

$$
\begin{aligned}
\frac{\partial P}{\partial x}= & 1-\frac{2}{k} x-\frac{y}{(1+x)^{2}}, \quad \frac{\partial Q}{\partial y}=\frac{b x}{1+x}+d\left(1-\frac{x}{k}\right)-m \\
\frac{\partial \alpha}{\partial x}= & -p, \quad \frac{\partial \alpha}{\partial y}=0, \quad \frac{\partial \beta}{\partial x}=0, \quad \frac{\partial \beta}{\partial y}=q, \quad \frac{\partial \phi}{\partial x}=1, \quad \frac{\partial \phi}{\partial y}=0 \\
\Delta_{1}= & \frac{P_{+}((\partial \beta / \partial y)(\partial \phi / \partial x)-(\partial \beta / \partial x)(\partial \phi / \partial y)+\partial \phi / \partial x)}{P(\partial \phi / \partial x)+Q(\partial \phi / \partial y)} \\
& +\frac{Q_{+}((\partial \alpha / \partial x)(\partial \phi / \partial y)-(\partial \alpha / \partial y)(\partial \phi / \partial x)+\partial \phi / \partial y)}{P(\partial \phi / \partial x)+Q(\partial \phi / \partial y)} \\
= & \frac{P^{+}\left(\xi\left(T^{+}\right), \eta\left(T^{+}\right)\right)(1+q)}{P(\xi(T), \eta(T))}=(1-p)(1+q) \frac{k-(1-p) h}{k-h}
\end{aligned}
$$


Furthermore,

$$
\begin{aligned}
\exp \left[\int_{0}^{T}\left(\frac{\partial P}{\partial x}(\xi(t), \eta(t))+\frac{\partial Q}{\partial y}(\xi(t), \eta(t))\right) d t\right] \\
\quad=\exp \left[\int_{0}^{T}\left(1-\frac{2}{k} \xi(t)+\frac{b \xi(t)}{1+\xi(t)}+d-m-\frac{d}{k} \xi(t)\right) d t\right] \\
=\left(\frac{k-(1-p) h}{(k-h)(1-p)}\right)^{1+d-m}\left(\frac{k-(1-p) h}{k-h}\right)^{-(2+d)}\left(\frac{(1+h)(k-(1-p) h)}{(k-h)(1+(1-p) h)}\right)^{b k /(1+k)}
\end{aligned}
$$

Therefore, it is possible to obtain the Floquet multiplier $\mu$ by direct calculation as follows:

$$
\begin{aligned}
\mu= & \prod_{k=1}^{n} \Delta_{k} \exp \left[\int_{0}^{T}\left(\frac{\partial P}{\partial x}(\xi(t), \eta(t))+\frac{\partial Q}{\partial y}(\xi(t), \eta(t))\right) d t\right] \\
= & (1-p)(1+q) \frac{k-(1-p) h}{k-h}\left(\frac{k-(1-p) h}{(k-h)(1-p)}\right)^{1+d-m}\left(\frac{k-(1-p) h}{k-h}\right)^{-(2+d)} \\
& \times\left(\frac{(1+h)(k-(1-p) h)}{(k-h)(1+(1-p) h)}\right)^{b k /(1+k)} \\
= & (1+q)(1-p)^{m-d}\left(\frac{k-(1-p) h}{k-h}\right)^{(b k /(1+k))-m}\left(\frac{1+h}{1+(1-p) h}\right)^{b k /(1+k)} .
\end{aligned}
$$

Hence, $|\mu|<1$ when (2.9) holds. This completes the proof.

Remark 2.4. When $q^{*}=(1-p)^{d-m}((k-(1-p) h) /(k-h))^{m-b k /(1+k)}((1+h) /(1+(1-p) h))^{-b k /(1+k)}$ -1 , a bifurcation may occur if $q=q^{*}$ for $|\mu|=1$, and a positive periodic solution may appear when $q>q^{*}$. Hence, the topic of bifurcation will be discussed in the following.

First, consider the Poincaré map (2.1), but with $\tau=0$. Set $u=y_{n}^{+}$and $u \geq 0$ small enough. Then the map becomes the following form:

$$
u \longmapsto(1+q) g(u) \equiv G(u, q)
$$

where the function $G(u, q)$ is continuously differentiable with respect to both $u$ and $q, g(0)=$ 0 ; then $\lim _{u \rightarrow 0^{+}} g(u)=g(0)=0$.

Second, from the bifurcation of map (2.14), the following theorem can be obtained.

Theorem 2.5. A transcritical bifurcation occurs when $q=q^{*}$. Therefore, a stable positive fixed point appears when the parameter $q$ changes through $q^{*}$ from left to right. Correspondingly, system (1.3) has a stable positive periodic solution if $q \in\left(q^{*}, q^{*}+\delta\right)$ with $\delta>0$. 
Proof. The values of $g^{\prime}(u)$ and $g^{\prime \prime}(u)$ must be calculated at $u=0$, where $0 \leq u \leq r(1-p) h(k-$ $(1-p) h) / k d \equiv u_{0}$; then system (1.3) can be transformed as follows:

$$
\frac{d y}{d x}=\frac{Q(x, y)}{P(x, y)}
$$

where

$$
P(x, y)=x\left(1-\frac{x}{k}\right)-\frac{x y}{(1+x)}, \quad Q(x, y)=\frac{b x y}{(1+x)}+d y\left(1-\frac{x}{k}\right)-m y .
$$

Let $\left(x, y\left(x ; x_{0}, y_{0}\right)\right)$ be an orbit of system (2.15) and set $x_{0}=(1-p) h, y_{0}=u, 0 \leq u \leq u_{0}$; then

$$
y(x ;(1-p) h, u) \equiv y(x, u), \quad(1-p) h \leq x \leq h, \quad 0 \leq u \leq u_{0} .
$$

Using (2.17),

$$
\begin{gathered}
\frac{\partial y(x, u)}{\partial u}=\exp \left[\int_{(1-p) h}^{x} \frac{\partial}{\partial y}\left(\frac{Q(s, y(s, u))}{P(s, y(s, u))}\right) d s\right] \\
\frac{\partial^{2} y(x, u)}{\partial u^{2}}=\frac{\partial y(x, u)}{\partial u} \int_{(1-p) h}^{x} \frac{\partial^{2}}{\partial y^{2}}\left(\frac{Q(s, y(s, u))}{P(s, y(s, u))}\right) \frac{\partial y(s, u)}{\partial u} d s .
\end{gathered}
$$

Clearly, it can be deduced that $\partial y(x, u) / \partial u>0$, and

$$
\begin{aligned}
g^{\prime}(0) & =\frac{\partial y(h, 0)}{\partial u}=\exp \left(\int_{(1-p) h}^{h} \frac{\partial}{\partial y}\left(\frac{Q(s, y(s, 0))}{P(s, y(s, 0))}\right) d s\right) \\
& =\exp \left(\int_{(1-p) h}^{h} \frac{b}{(1+s)(1-s / k)}+\frac{d}{s}-\frac{m}{s(1-s / k)} d s\right) \\
& =(1-p)^{m-d}\left(\frac{k-(1-p) h}{k-h}\right)^{k b /(k+1)-m}\left(\frac{1+h}{1+(1-p) h}\right)^{k b /(k+1)} .
\end{aligned}
$$

Furthermore,

$$
g^{\prime \prime}(0)=g^{\prime}(0) \int_{(1-p) h}^{h} m(s) \frac{\partial y(s, 0)}{\partial u} d s
$$

where

$$
\begin{array}{r}
m(s)=\frac{\partial^{2}}{\partial y^{2}}\left(\frac{Q(s, y(s, 0))}{P(s, y(s, 0))}\right)=\frac{s}{(1+s)(s(1-s / k))^{2}}\left(\frac{b s}{1+s}+d\left(1-\frac{s}{k}\right)-m\right), \\
s \in[(1-p) h, h] .
\end{array}
$$


Using the previous assumption $d y<0$ in $\Omega$ yields $b s /(1+s)+d(1-s / k)-m<0$. It can be determined that $m(s)<0, s \in[(1-p) h, h)$.

Therefore,

$$
g^{\prime \prime}(0)<0
$$

The next step is to check whether the following conditions are satisfied.

(a) It is easy to see that

$$
G(0, q)=0, \quad q \in(0, \infty)
$$

(b) Using (2.19),

$$
\frac{\partial G(0, q)}{\partial u}=(1+q) g^{\prime}(0)=(1+q)(1-p)^{m-d}\left(\frac{k-(1-p) h}{k-h}\right)^{(k b /(k+1))-m}\left(\frac{1+h}{1+(1-p) h}\right)^{k b /(k+1)}
$$

which yields

$$
\frac{\partial G\left(0, q^{*}\right)}{\partial u}=1
$$

This means that $\left(0, q^{*}\right)$ is a fixed point with eigenvalue 1 of map (2.14).

(c) Because (2.19) holds,

$$
\frac{\partial^{2} G\left(0, q^{*}\right)}{\partial u \partial q}=g^{\prime}(0)>0
$$

(d) Finally, inequality (2.22) implies that

$$
\frac{\partial^{2} G\left(0, q^{*}\right)}{\partial u^{2}}=\left(1+q^{*}\right) g^{\prime \prime}(0)<0
$$

These conditions satisfy the conditions of Lemma 2.2. This completes the proof.

\subsection{Case $\tau>0$}

This subsection discusses the existence of a positive periodic solution with $\tau>0$ using the Poincaré map (2.2).

Case 1. $h<x_{2}^{*}$.

Theorem 2.6. For any $q>0$ and $\tau>0$, system (1.3) has a positive period-1 solution. 
Proof. Choose a point $A_{1}((1-p) h, \varepsilon)$ from section $S_{0}$, where $\varepsilon(\varepsilon<\tau)$ is small enough. The trajectory starting with initial point $A_{1}$ intersects section $S_{1}$ at point $B_{1}\left(h, \varepsilon_{0}\right)$. Because of impulsive effects, the trajectory jumps to point $A_{2}\left((1-p) h,(1+q) \varepsilon_{0}+\tau\right)$ on section $S_{0}$ and then returns to point $B_{2}\left(h, \varepsilon_{2}\right)$ on $S_{1}$. For $(1+q) \varepsilon_{0}+\tau>\varepsilon$, point $A_{2}$ is above point $A_{1}$. Furthermore, point $B_{2}$ is above point $B_{1}$, and $\varepsilon_{2}>\varepsilon_{0}$. Hence, from $(2.2), \varepsilon_{2}=F\left(q, \tau, \varepsilon_{0}\right)$, and furthermore,

$$
\varepsilon_{0}-F\left(q, \tau, \varepsilon_{0}\right)=\varepsilon_{0}-\varepsilon_{2}<0 .
$$

Next, assume that the horizontal isocline $l: y=(1-x / k)(1+x)$ intersects the Poincare section $S_{0}$ at $W((1-p) h,(1-(1-p) h / k)(1+(1-p) h))$. The trajectory from the initial point $W$ intersects the Poincaré section $S_{1}$ at point $V\left(h, v_{0}\right)$ jumps to point $V^{+}\left((1-p) h,(1+q) v_{0}+\tau\right)$, and then returns to point $V_{1}\left(h, v_{1}\right)$ on section $S_{1}$. Assume that there exists a $q_{0}>0$ such that $\left(1+q_{0}\right) v_{0}+\tau=(1-(1-p) h / k)(1+(1-p) h)$; then point $V^{+}$coincides with point $W$ only for $q=q_{0}$, which is above point $W$ for $q>q_{0}$, while it is below point $W$ for $q<q_{0}$. However, for any $q>0$, point $V_{1}$ is not above point $V$ because of the properties of the vector field of system (1.3), which means that $v_{1} \leq v_{0}$.

(a) If $v_{1}=v_{0}$, then system (1.3) has a positive period-1 solution;

(b) If $v_{1}<v_{0}$, then

$$
v_{0}-F\left(q, \tau, v_{0}\right)=v_{0}-v_{1}>0
$$

It is easy to see that the Poincaré map (2.2) has a fixed point by (2.28) and (2.29), or in other words, system (1.3) has a positive period-1 solution. This completes the proof.

Now the stability of this positive period-1 solution of system (1.3) will be discussed.

Theorem 2.7. For any $q>0, \tau>0$, let $(\xi(t), \eta(t))$ be a positive order-1 T-periodic solution of system (1.3) which starts from the point $(h, \omega)$. If the condition

$$
|\mu|=(1+q) \Gamma \exp \left(\int_{0}^{T} \Psi(t) d t\right)<1
$$

holds, where

$$
\Gamma=(1-p) \frac{1-(1-p) h / k-((1+q) \omega+\tau) /(1+(1-p) h)}{1-h / k-\omega /(1+h)}
$$

then $(\xi(t), \eta(t))$ is orbitally asymptotically stable.

Proof. Assume that the periodic solution with period $T$ passes through the points $K^{+}((1-$ $p) h,(1+q) \omega+\tau)$ and $K(h, \omega)$ in which $\omega \leq v_{1}$ holds. Because the form and the period $T$ of the solution are not known, the stability of this positive periodic solution will be discussed using Lemma 2.1. The difference between this case and that of Theorem 2.3 lies in that

$$
(\xi(T), \eta(T))=(h, \omega), \quad\left(\xi\left(T^{+}\right), \eta\left(T^{+}\right)\right)=((1-p) h,(1+q) \omega+\tau),
$$


while other factors are the same. Then,

$$
\begin{aligned}
\Delta_{1}= & \frac{P_{+}((\partial \beta / \partial y)(\partial \phi / \partial x)-(\partial \beta / \partial x)(\partial \phi / \partial y)+\partial \phi / \partial x)}{P(\partial \phi / \partial x)+Q(\partial \phi / \partial y)} \\
& +\frac{Q_{+}((\partial \alpha / \partial x)(\partial \phi / \partial y)-(\partial \alpha / \partial y)(\partial \phi / \partial x)+\partial \phi / \partial y)}{P(\partial \phi / \partial x)+Q(\partial \phi / \partial y)} \\
= & \frac{P^{+}\left(\xi\left(T^{+}\right), \eta\left(T^{+}\right)\right)(1+q)}{P(\xi(T), \eta(T))}=(1+q) \Gamma
\end{aligned}
$$

where

$$
\Gamma=(1-p) \frac{1-(1-p) h / k-((1+q) \omega+\tau) / 1+((1-p) h)}{1-h / k-\omega /(1+h)} .
$$

Let $\Psi(t)=(\partial P / \partial x)(\xi(t), \eta(t))+(\partial Q / \partial y)(\xi(t), \eta(t))$; then

$$
\begin{aligned}
|\mu| & =\Delta_{1} \exp \left(\int_{0}^{T}\left(\frac{\partial P}{\partial x}(\xi(t), \eta(t))+\frac{\partial Q}{\partial y}(\xi(t), \eta(t))\right) d t\right) \\
& =(1+q) \Gamma \exp \left(\int_{0}^{T} \Psi(t) d t\right) .
\end{aligned}
$$

If $|\mu|<1$, that is,

$$
\left|(1+q) \Gamma \exp \left(\int_{0}^{T} \Psi(t) d t\right)\right|<1,
$$

then the periodic solution is stable. This completes the proof.

Remark 2.8. From the above, it is apparent that if there exists a $q^{\prime}>q_{0}$ such that $|u|=1$, a flip bifurcation occurs at $q=q^{\prime}$. If a flip bifurcation occurs, there exists a stable positive period-2 solution of system (1.3) for $q^{\prime}>q_{0}$, which may also lose its stability when $q$ increases.

Case $2\left((1-p) h<x_{2}^{*}<h<x_{1}^{*}\right)$. In this case, the following theorem can be proved.

Theorem 2.9. There exists a $\tau_{0}=g(h)>0$ such that for any $\tau>\tau_{0}$ and $q>0$, system (1.3) has a positive period-1 or period-2 solution which has asymptotic orbital stability. Furthermore, system (1.3) has no period- $k$ solution $(k \geq 3)$.

Proof. The phase diagram of system (1.3) without impulses is shown in Figure 1(a). Assume that the trajectory crosses $S_{0}$ at points $A\left((1-p) h, y_{1}\right)$ and $A_{1}\left((1-p) h, y_{2}\right)$, respectively, where $y_{2}<(1-(1-p) h / k)(1+(1-p) h)<y_{1}$, and is tangent to $S_{1}$ at point $B\left(h, y_{3}\right), y_{3}=(1-h / k)(1+h)$. For any $y \in\left(y_{2}, y_{1}\right)$, the trajectory of system (1.3) without impulsive passing through point $((1-p) h, y)$ will not intersect with $S_{0}$ over time. Eventually, it will tend to the focus $\left(x_{2}^{*}, y_{2}^{*}\right)$. 
Hence, after several impulsive actions on $S_{1}$, the trajectory of system (1.3) that passed through points $((1-p) h, y)\left(y \in\left(y_{2}, y_{1}\right)\right)$ will eventually tend to the focus, and then there is no positive periodic solution (Figure 1(b)). Therefore, $\tau>y_{1}$ is a sufficient condition for a trajectory of system (1.3) to intersect with $S_{1}$ an infinite number of times due to impulsive effects, where $y_{1}=g(h)$ depends on the threshold value $h$.

Therefore, for any two points $G_{i}\left(h, g_{i}\right)$ and $D_{j}\left(h, d_{j}\right)$ at which $0<g_{i}<d_{j}<(1-$ $h / k)(1+h)$, the points $G_{i}^{+}\left((1-p) h,(1+q) g_{i}+\tau\right)$ and $D_{j}^{+}\left((1-p) h,(1+q) d_{j}+\tau\right)$ are above the point $A$. Then it follows from the vector field of system (1.3) without impulsive that $0<d_{j+1}<g_{i+1}<(1-h / k)(1+h)$, that is,

$$
g_{i+1}>d_{j+1} \quad \text { for } 0<g_{i}<d_{j}<\left(1-\frac{h}{k}\right)(1+h), \quad \tau>y_{1}>(1+(1-p) h)\left(1-\frac{(1-p) h}{k}\right) .
$$

Now, for any $y_{0} \in(0,(1+h)(1-h / k)), y_{1}=F\left(q, \tau, y_{0}\right), y_{2}=F\left(q, \tau, y_{1}\right)$, and $y_{n+1}=F\left(q, \tau, y_{n}\right)$ $(n=3,4, \ldots)$ by the Poincaré map (2.2). If $y_{0}=y_{1}$, then system (1.3) has a positive period-1 solution. If $y_{0} \neq y_{1}$ and $y_{0}=y_{2}$, system (1.3) has a positive period-2 solution.

Next, the general case will be discussed, that is, $y_{0} \neq y_{1} \neq y_{2} \neq \cdots \neq y_{k-1}(k \geq 3)$ and $y_{0}=y_{k}$. Then system (1.3) has a positive period- $k$ solution. In fact, this is impossible.

Case $1\left(y_{0}<y_{1}\right)$. From (2.37), $y_{1}>y_{2}$. Therefore, the relationship among $y_{0}, y_{1}, y_{2}$ is either $y_{1}>y_{0}>y_{2}$ or $y_{1}>y_{2}>y_{0}$.

(1) $y_{1}>y_{0}>y_{2}$ : if $y_{1}>y_{0}>y_{2}$, then from (2.37), $y_{3}>y_{1}>y_{2}$. It is also true that $y_{3}>y_{1}>y_{0}>y_{2}$. Repeating this process yields:

$$
0<\cdots<y_{2 k}<\cdots<y_{4}<y_{2}<y_{0}<y_{1}<y_{3}<\cdots<y_{2 k+1}<\cdots<\left(1-\frac{(1-p) h}{k}\right)(1+h)
$$

(2) $y_{1}>y_{2}>y_{0}$ : following the same argument, if $y_{1}>y_{2}>y_{0}$,

$$
0<y_{0}<y_{2}<y_{4} \cdots<y_{2 k}<\cdots<y_{2 k+1}<\cdots<y_{3}<y_{1}<\left(1-\frac{(1-p) h}{k}\right)(1+h)
$$

Case $2\left(y_{0}>y_{1}\right)$. In this case, the relationship among $y_{0}, y_{1}, y_{2}$ has two types as well: $y_{0}>$ $y_{2}>y_{1}$ or $y_{2}>y_{0}>y_{1}$.

(1) $y_{0}>y_{2}>y_{1}$ : if $y_{0}>y_{2}>y_{1}$, then

$$
0<y_{1}<y_{3}<y_{5}<\cdots<y_{2 k+1}<\cdots<y_{2 k}<\cdots<y_{4}<y_{2}<y_{0}<\left(1-\frac{(1-p) h}{k}\right)(1+h) .
$$




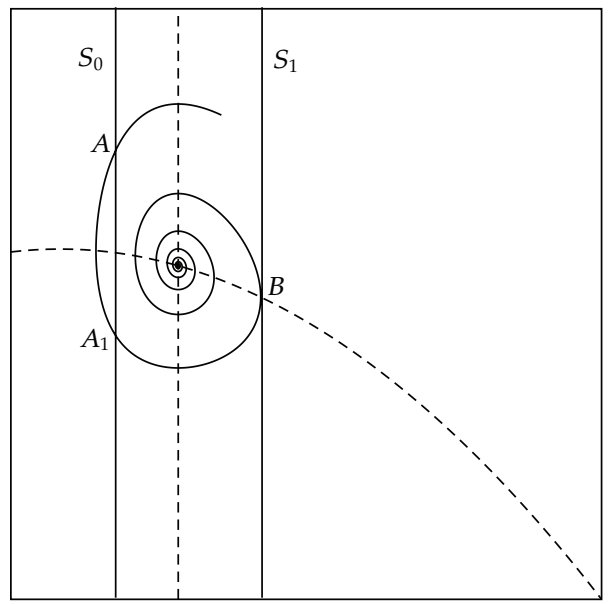

(a)

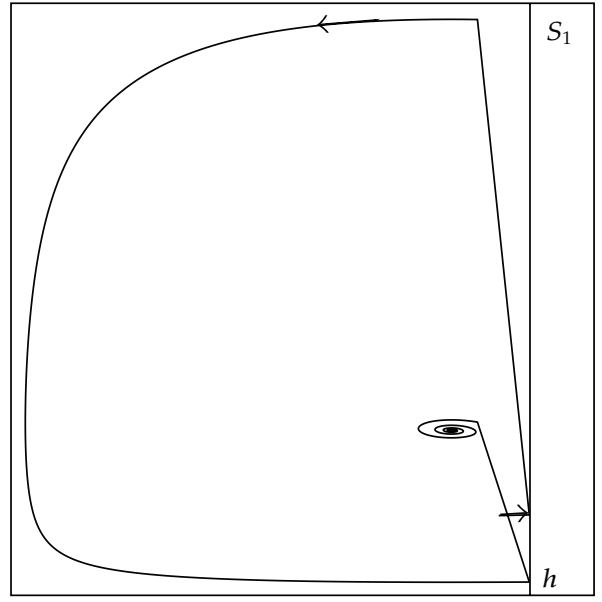

(b)

Figure 1: (a) Phase diagram of system (1.3) without impulses; (b) phase diagram of system (1.3).

(2) $y_{2}>y_{0}>y_{1}:$ if $y_{2}>y_{0}>y_{1}$, then

$0<\cdots<y_{2 k+1}<\cdots<y_{3}<y_{1}<y_{0}<y_{2}<y_{4}<\cdots<y_{2 k}<\cdots<\left(1-\frac{(1-p) h}{k}\right)(1+h)$

If system (1.3) has a period- $k$ solution $(k \geq 3)$, then $y_{0} \neq y_{1} \neq y_{2} \neq \cdots \neq y_{k-1}(k \geq 3)$, $y_{0}=y_{k}$, which by Case 1 and Case 2, is a contradiction. Therefore, system (1.3) has no period$k(k \geq 3)$ solution. However, there is a stable period-1 or period-2 solution in this case. In Case 1 of (1), $\lim _{n \rightarrow+\infty} y_{2 k}=y^{*}$ and $\lim _{n \rightarrow+\infty} y_{2 k+1}=y^{+}$, where $0<y^{*}<y^{+}<(1+h)(1-h / k)$. Hence, $y^{*}=f\left(q, y^{+}\right)$and $y^{+}=f\left(q, y^{*}\right)$. Therefore, system (1.3) has a stable period-2 solution in this case. In similar manner, it can be determined that system (1.3) has a stable period-1 solution in Case 1 of (2) and Case 2 of (1). In Case 2 of (2), system (1.3) has a stable period-2 solution. In the above proof, $\tau>y_{1}$. This completes the proof.

By using the theoretical analysis, we obtain the threshold expression of some critical parameters under the condition of the existence and stability of semi-trivial periodic solutions as well as the transcritical bifurcation, which in turn provides a theoretical basis for the numerical simulation.

\section{Numerical Analysis}

It is known that the continuous system corresponding to system (1.3) cannot be solved explicitly, so system (1.3) must be investigated using numerical simulation. In this section, the parameters are fixed as: $k=1.2, b=1.8, d=0.2, m=0.6$.

By direct calculation, $+b-d / k-m \approx 0.226>0, m-d=0.4>0,(d+b-d / k-m)^{2}=$ $1.521>0.267 \approx(4 d / k)(m-d), x_{2}^{*} \approx 0.34, y_{2}^{*} \approx 0.96$, and $\left(1+2 x_{2}^{*}\right) / k \approx 1.4>1$, and therefore the interior equilibrium point $P_{2}^{*}\left(x_{2}^{*}, y_{2}^{*}\right)$ is a stable positive focus, as shown in Figure 2. 


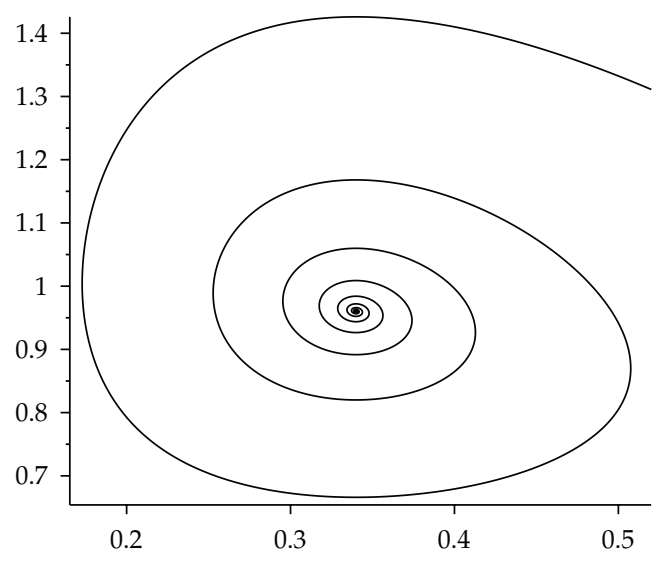

(a)

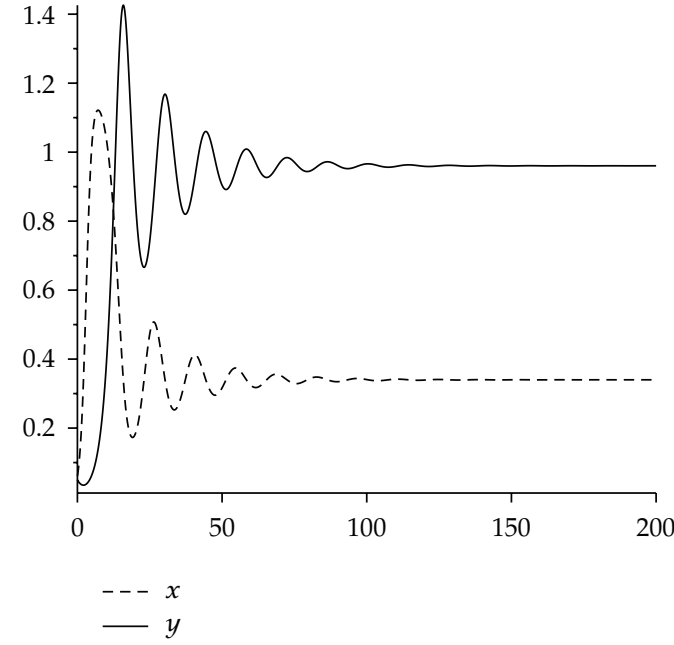

(b)

Figure 2: (a) Phase diagram of system (1.3) without impulses; (b) time series in $x$ and $y$ of system (1.3) without impulsive.

For $\tau=0$, it is known that a semi-trivial periodic solution exists for system (1.3); furthermore, it is stable when (2.9) holds. Set $p=0.6, h=0.2$; by Remark 2.4, $q^{*} \approx 0.246$. Let $q=0.18$; then the semi-trivial periodic solution is stable, and the solution from the initial point $(0.06,0.05)$ of system $(1.3)$ tends to the stable semi-trivial periodic solution as $t$ increases (Figure 3(a)). Now let $q=0.5$; it is easy to see in Figure 3(b) that the semi-trivial periodic solution has become unstable.

In the case of $h<x_{2}^{*}$, from Theorems 2.6 and 2.5, there exists a stable positive period-1 solution of system (1.3) for any $q>0$ and $\tau>0$. Furthermore, from Remark 2.8, there may be a positive period $i(i>1)$ which leads to the loss of stability as $q$ increases, as shown in Figures 4 and 5 .

In fact, it is obvious that for system (1.3), there exists a positive periodic solution for any $q>0$ and $\tau>0$ or $q \in\left(q^{*}, q^{*}+\delta\right)$ with $\delta>0$ and $\tau=0$ in Figures 6(a), 6(b), and 6(c). Figure 6(a) shows the bifurcation diagram of system (1.3) plotted as a function of $q$ in the case of $\tau=0$; the bifurcation diagram of system (1.3) with $\tau>0$ is shown in Figure 6(b). On the other hand, viewing $\tau$ as a bifurcation parameter in system (1.3), a different bifurcation diagram for system (1.3) is obtained (Figure 6(c)).

In Figure 6(a), 6(b), and 6(c), it is easy to see that system (1.3) shows rich population dynamic behavior consistent with the theoretical analysis, such as period-doubling bifurcations, a chaotic band, a periodic window, chaotic crises, period-halving bifurcations, and so on. In Figure 6(a), it is obvious that there exists a semi-trivial solution which is stable for $q \in(0,0.64)$, which implies that the population $P$ goes extinction because of the fixed release amount $\tau=0$. This result suggests that the value of the fixed release amount $\tau$ can affect the coexistence of the population $N$ and the population $P$. A fold bifurcation occurs at $q=0.64$, where a positive period- 1 solution bifurcates from the periodic semitrivial solution. Furthermore, a positive period-2 solution bifurcates from the positive period1 solution through a flip bifurcation at $q=3.92$, while the positive period-1 solution is stable for $q \in(0.64,3.92)$. Finally, the period-doubling bifurcation leads to chaos. Furthermore, it 


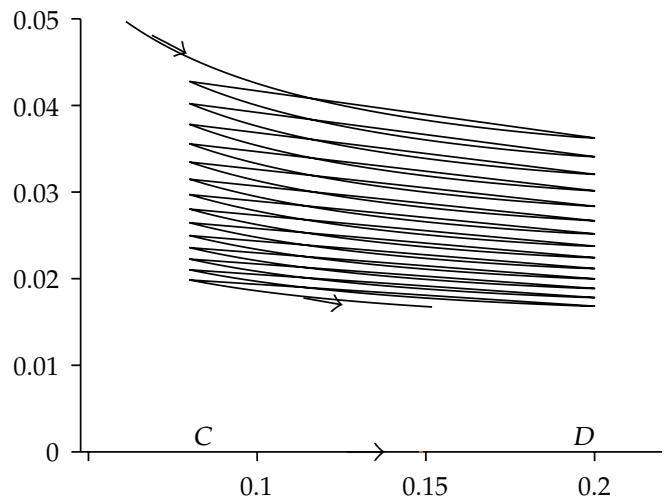

(a)

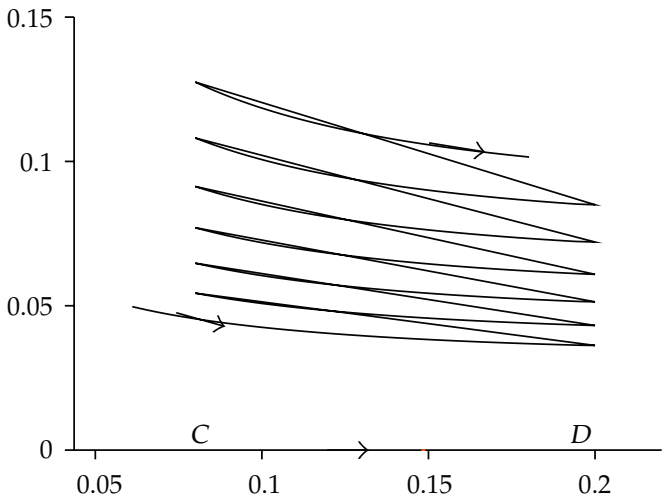

(b)

Figure 3: Trajectories with initial point $(0.06,0.05)$ of system $(1.3)$ with $p=0.6, h=0.2$ (a) $q=0.18$, (b) $q=0.5$.

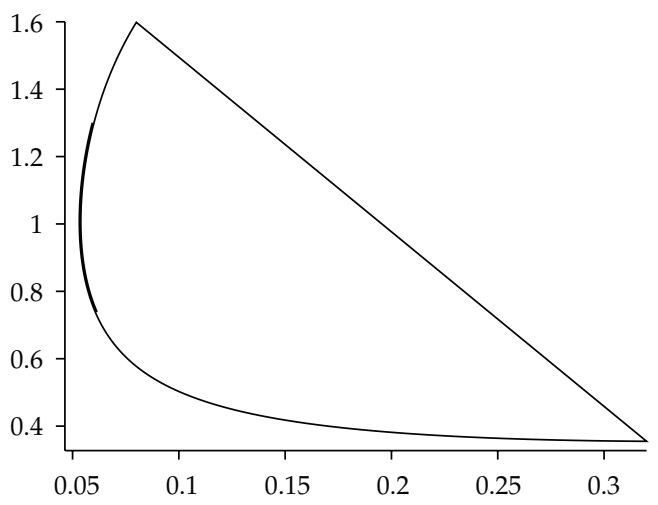

(a)

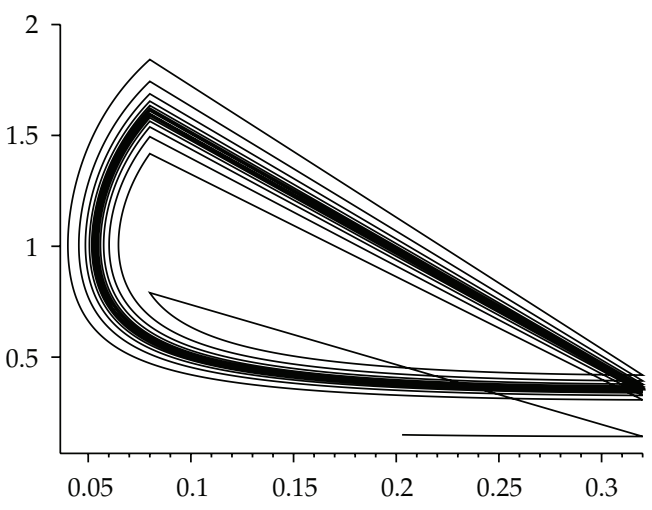

(b)

Figure 4: (a) Existence of positive period-1 solution; (b) stability of positive period-1 solution.

is interesting to find from Figure 6(a) that the max amount of the population $P$ gradually increases as the value of $q$ increases from 0.64 to 30 . These results indicate that the fixed release amount $\tau$ has a positive impact for the population $P$ persistence and biological diversity in real ecological communities. Nonetheless, it is worthwhile to notice that the max amount of population $P$ has not been greatly affected.

When $\tau>0$, as shown in Figure 6(b), there is no periodic semi-trivial solution of system (1.3), and there exists no fold bifurcation. However, there is a positive period-1 solution, which is stable for $q \in(0,4.42)$, which implies that a low value of the fixed release amount $\tau$ can promote the population $P$ persistence. A period-2 solution appears due to loss of stability at $q=4.42$. Subsequently, a series of periodic-doubling bifurcations leads to chaotic solutions. A periodic-halving bifurcation leads to period-3 solutions for $q>33.64$. According to Li and Yorke theorem, period-3 implies chaos, and chaotic solutions will appear as $q$ increases. Comparing Figure 6(a) with Figure 6(b), it is evident to be found that the system (1.3) has different dynamical behaviors when the value of the fixed release amount $\tau$ is 0 or 0.0175 . On the other hand, in Figure 6(c), there is a route from chaotic solutions to 


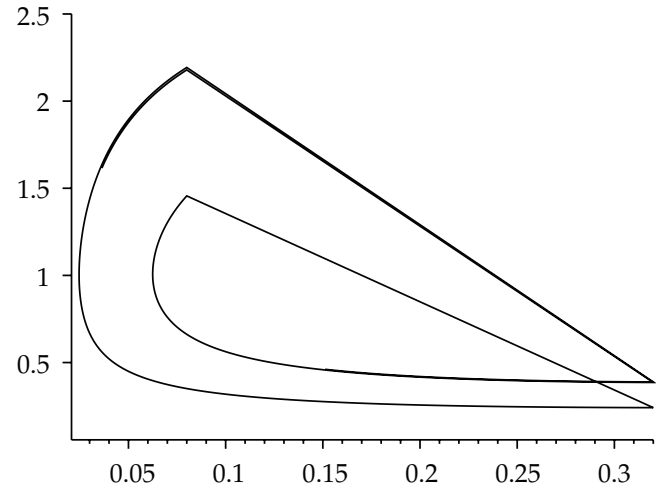

(a)

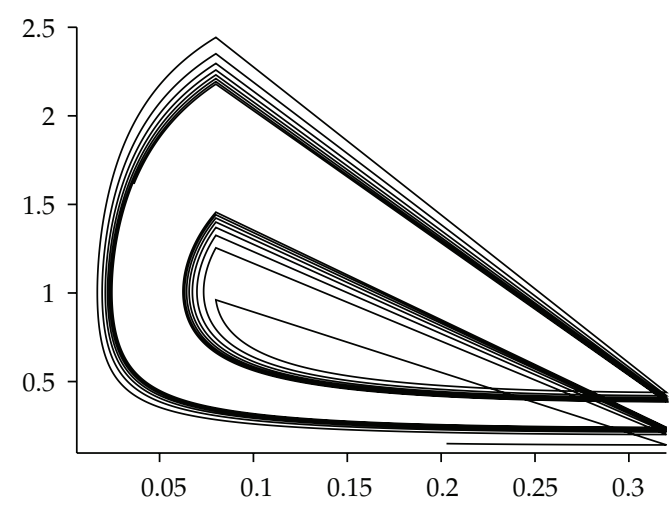

(b)

Figure 5: (a) Existence of positive period-2 solution; (b) stability of positive period-2 solution.

stable periodic solutions through a cascade of period-halving bifurcations. Hence, it should be stressed that the fixed release amount $\tau$ can not only promote population persistence, but also affect complex population dynamic in the predator-prey system (1.3).

In the case of $h>x_{2}^{*}$, from Theorem 2.9, there exists some $\tau_{0}>0$ such that system (1.3) has a stable positive period-1 or period-2 solution for $\tau>\tau_{0}$ and any $q>0$. Let $h=0.5$ and $\tau=0.8$; this leads to a different bifurcation diagram of system (1.3) about the bifurcation parameter $q$, which is shown in Figure 6(d). It is easy to see that stable positive period-1 and period-2 solutions exist, but that there is no period- $k(k=3,4 \ldots)$ solution. Assuming $q=4$, Figure 7 shows the phase diagram of a stable periodic solution and a time series in $y$ for system (1.3). These results show that the large values of the fixed release amount $\tau$ can suppress the emergence of chaos, and thus it is interesting to observe that the large values of the fixed release amount $\tau$ have an important effect on the system stability under the condition of $(1-p) h<x_{2}^{*}<h<x_{1}^{*}$. Furthermore, when the value of $h$ is different, the system (1.3) has completely different dynamical behaviors.

Based on the above analysis, it is obvious that numerical results are consistent with mathematical theoretical works. Moreover, it is also successful for impulsive state feedback control strategy to maintain two species persistence, and thus it is worthwhile to point out that the key factors for long-term complex dynamics of the system (1.3) are impulsive state feedback control strategy, especially the fixed release amount $\tau$.

\section{Conclusions}

In this research, a predator-prey model with impulsive state feedback control was built and studied analytically and numerically. Mathematical theoretical investigations have addressed the existence and stability of semi-trivial periodic solutions of system (1.3) and have proved that positive periodic solutions come into being from semi-trivial periodic solutions through a transcritical bifurcation, as described by bifurcation theory. These mathematical works in turn provide a theoretical basis for the numerical simulation.

Numerical simulations indicates that complex population dynamics of the system (1.3) depend on the parameters of impulsive state feedback control strategy and the controlling threshold for the population $N$. With this framework, the direct and indirect effects on population persistence and dynamical behavior of the system (1.3) caused by impulsive 


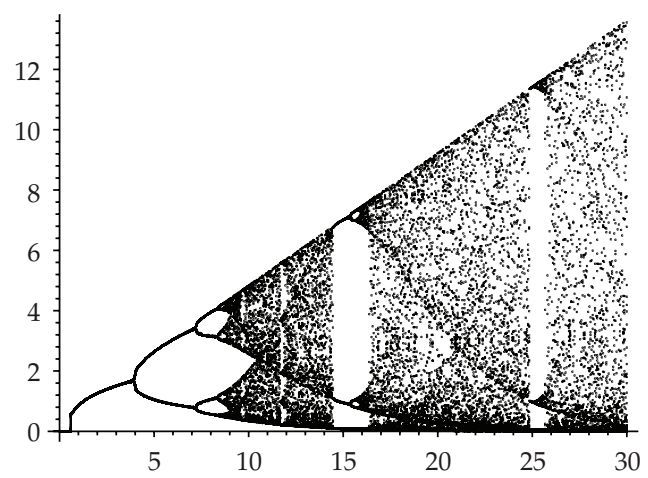

(a)

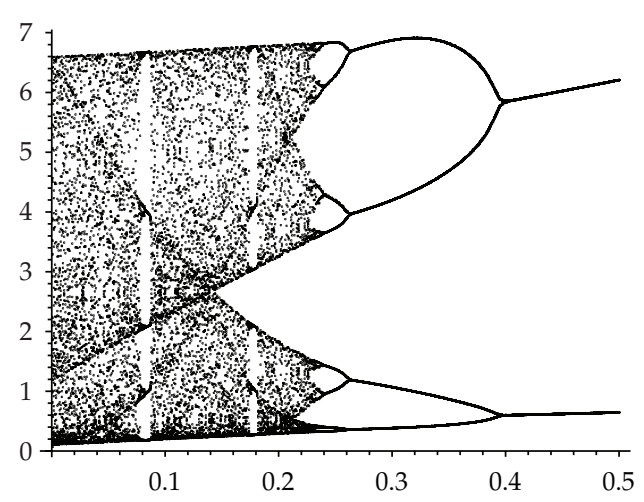

(c)

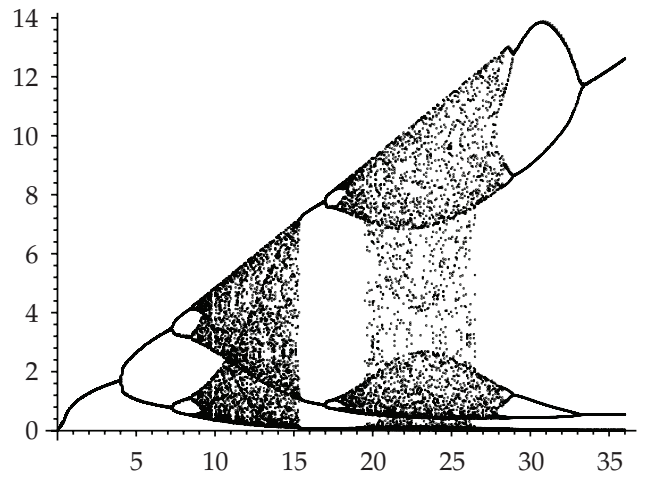

(b)

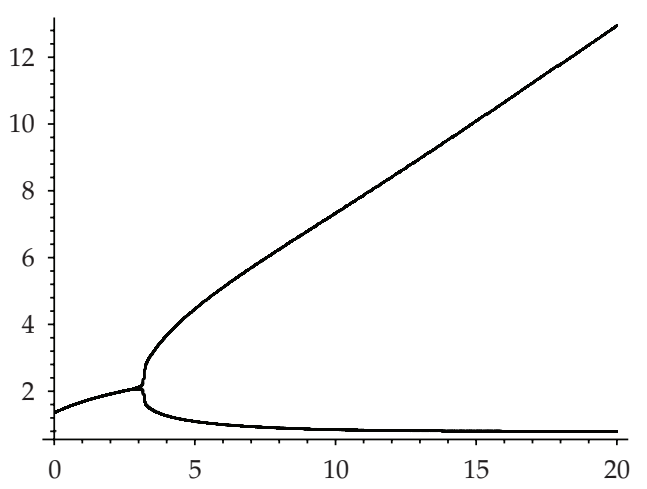

(d)

Figure 6: Bifurcation diagram of system (1.3) with $p=0.75$, (a) $h=0.32, \tau=0$, (b) $h=0.32, \tau=0.0175$, (c) $h=0.32, q=14$, (d) $h=0.5, \tau=0.8$.

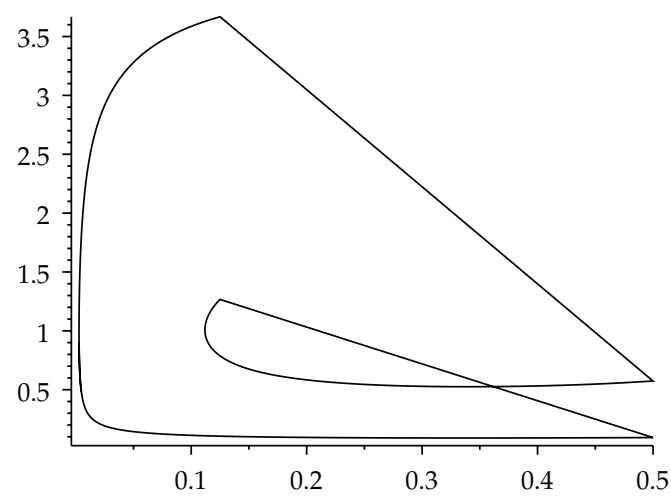

(a)

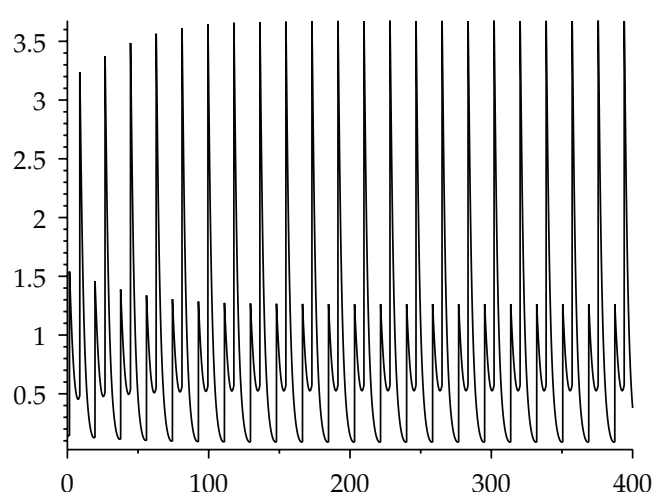

(b)

Figure 7: Periodic solution of system (1.3) with $p=0.75, h=0.5, \tau=0.8$. (a) Phase diagram, (b) time series of $y$. 
state feedback control strategy and the controlling threshold for the population $N$ are investigated by means of bifurcation analysis. It should be stressed that if there is not the fixed release amount $\tau$ for the population $P$, the population $P$ is not permanent for $q \in(0,0.64)$, which implies a stable semi-trivial solution, but the low values of the fixed release amount $\tau$ have a positive effect on the population persistence. Whatsoever, the large values of the controlling threshold and the fixed release amount $\tau$ have a profound effect on the population stability under the condition of other fixed parameters. It is worthwhile to remark that the values of the fixed release amount $\tau$ have no negative effect on the biomass of the population. In a word, impulsive state feedback control strategy can alter population dynamics affecting the interaction strength among population, increasing strong links according to population feature, which in turn illustrates that the impulsive control strategy is feasible and meaningful.

These results have important implications for conservation management, especially endangered biological species, and are expected to be of use in the study of the dynamic complexity of ecosystems.

\section{Acknowledgments}

The authors would like to thank the editor and the anonymous referees for their valuable comments and suggestions on this paper. This work was supported by the National Natural Science Foundation of China (Grant no. 31170338 and Grant no. 30970305).

\section{References}

[1] G. R. Jiang and Q. S. Lu, "Impulsive state feedback control of a predator-prey model," Journal of Computational and Applied Mathematics, vol. 200, no. 1, pp. 193-207, 2007.

[2] L. Nie, Z. Teng, L. Hu, and J. Peng, "The dynamics of a Lotka-Volterra predator-prey model with state dependent impulsive harvest for predator," BioSystems, vol. 98, no. 2, pp. 67-72, 2009.

[3] V. Lakshmikantham, D. D. Baĭnov, and P. S. Simeonov, Theory of Impulsive Differential Equations, World Scientific, Singapore, 1989.

[4] H. Yu, S. Zhong, R. P. Agarwal, and S. K. Sen, "Three-species food web model with impulsive control strategy and chaos," Communications in Nonlinear Science and Numerical Simulation, vol. 16, no. 2, pp. 1002-1013, 2011.

[5] W. B. Wang, J. H. Shen, and J. J. Nieto, "Permanence and periodic solution of predator-prey system with holling type functional response and impulses," Discrete Dynamics in Nature and Society, vol. 2007, Article ID 81756, 15 pages, 2007.

[6] H. Yu, S. Zhong, and R. P. Agarwal, "Mathematics and dynamic analysis of an apparent competition community model with impulsive effect," Mathematical and Computer Modelling, vol. 52, no. 1-2, pp. 25-36, 2010.

[7] R. Q. Shi and L. S. Chen, "Stage-structured impulsive SI model for pest management," Discrete Dynamics in Nature and Society, vol. 2007, Article ID 97608, 11 pages, 2007.

[8] H. Yu, S. Zhong, R. P. Agarwal, and S. K. Sen, "Effect of seasonality on the dynamical behavior of an ecological system with impulsive control strategy," Journal of the Franklin Institute, vol. 348, no. 4, pp. 652-670, 2011.

[9] C. J. Wei and L. S. Chen, "A delayed epidemic model with pulse vaccination," Discrete Dynamics in Nature and Society, vol. 2008, Article ID 746951, 12 pages, 2008.

[10] H. Yu, S. Zhong, R. P. Agarwal, and L. Xiong, "Species permanence and dynamical behavior analysis of an impulsively controlled ecological system with distributed time delay," Computers $\mathcal{E}$ Mathematics with Applications, vol. 59, no. 12, pp. 3824-3835, 2010.

[11] H. Yu, S. Zhong, and R. P. Agarwal, "Mathematics analysis and chaos in an ecological model with an impulsive control strategy," Communications in Nonlinear Science and Numerical Simulation, vol. 16, no. 2, pp. 776-786, 2011. 
[12] Z. Teng, L. Nie, and X. Fang, "The periodic solutions for general periodic impulsive population systems of functional differential equations and its applications," Computers $\mathcal{E}$ Mathematics with Applications, vol. 61, no. 9, pp. 2690-2703, 2011.

[13] S. Lv and M. Zhao, "The dynamic complexity of a three species food chain model," Chaos, Solitons and Fractals, vol. 37, no. 5, pp. 1469-1480, 2008.

[14] J. Guckenheimer and P. Holmes, Nonlinear Oscillations, Dynamical Systems, and Bifurcations of Vector Fields, vol. 42, Springer, New York, NY, USA, 1983.

[15] L. Zhang and M. Zhao, "Dynamic complexities in a hyperparasitic system with prolonged diapause for host," Chaos, Solitons and Fractals, vol. 42, no. 2, pp. 1136-1142, 2009.

[16] R. I. Leine, D. H. Van Campen, and B. L. Van De Vrande, "Bifurcations in nonlinear discontinuous systems," Nonlinear Dynamics, vol. 23, no. 2, pp. 105-164, 2000.

[17] M. Zhao, L. Zhang, and J. Zhu, "Dynamics of a host-parasitoid model with prolonged diapause for parasitoid," Communications in Nonlinear Science and Numerical Simulation, vol. 16, no. 1, pp. 455-462, 2011.

[18] M. Zhao and S. Lv, "Chaos in a three-species food chain model with a Beddington-DeAngelis functional response," Chaos, Solitons and Fractals, vol. 40, no. 5, pp. 2305-2316, 2009.

[19] A. Lakmeche and O. Arino, "Bifurcation of non trivial periodic solutions of impulsive differential equations arising chemotherapeutic treatment," Dynamics of Continuous, Discrete and Impulsive Systems, vol. 7, no. 2, pp. 265-287, 2000.

[20] S. Y. Tang and L. S. Chen, "Density-dependent birth rate, birth pulses and their population dynamic consequences," Journal of Mathematical Biology, vol. 44, no. 2, pp. 185-199, 2002.

[21] G. Zeng, L. Chen, and L. Sun, "Existence of periodic solution of order one of planar impulsive autonomous system," Journal of Computational and Applied Mathematics, vol. 186, no. 2, pp. 466-481, 2006.

[22] L. Nie, Z. Teng, L. Hu, and J. Peng, "Qualitative analysis of a modified Leslie-Gower and Holling-type II predator-prey model with state dependent impulsive effects," Nonlinear Analysis, vol. 11, no. 3, pp. 1364-1373, 2010.

[23] L. Nie, J. Peng, Z. Teng, and L. Hu, "Existence and stability of periodic solution of a Lotka-Volterra predator-prey model with state dependent impulsive effects," Journal of Computational and Applied Mathematics, vol. 224, no. 2, pp. 544-555, 2009.

[24] L. Qian, Q. Lu, Q. Meng, and Z. Feng, “Dynamical behaviors of a prey-predator system with impulsive control," Journal of Mathematical Analysis and Applications, vol. 363, no. 1, pp. 345-356, 2010.

[25] S. Y. Tang and L. S. Chen, "Modelling and analysis of integrated pest management strategy," Discrete and Continuous Dynamical Systems B, vol. 4, no. 3, pp. 761-770, 2004.

[26] S. Y. Tang and R. A. Cheke, "State-dependent impulsive models of integrated pest management (IPM) strategies and their dynamic consequences," Journal of Mathematical Biology, vol. 50, no. 3, pp. 257-292, 2005.

[27] T. K. Kar and S. K. Chattopadhyay, "A focus on long-run sustainability of a harvested prey predator system in the presence of alternative prey," Comptes Rendus, vol. 333, no. 11-12, pp. 841-849, 2010.

[28] G. R. Jiang, Q. S. Lu, and L. M. Qian, "Complex dynamics of a Holling type II prey-predator system with state feedback control," Chaos, Solitons and Fractals, vol. 31, no. 2, pp. 448-461, 2007.

[29] P. S. Simeonov and D. D. BaĬnov, "Orbital stability of periodic solutions of autonomous systems with impulse effect," International Journal of Systems Science, vol. 19, no. 12, pp. 2561-2585, 1988.

[30] S. N. Rasband, Chaotic Dynamics of Nonlinear Systems, John Wiley \& Sons, New York, NY, USA, 1990. 


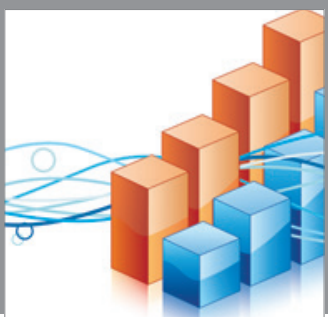

Advances in

Operations Research

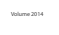

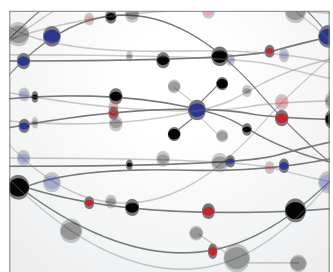

\section{The Scientific} World Journal
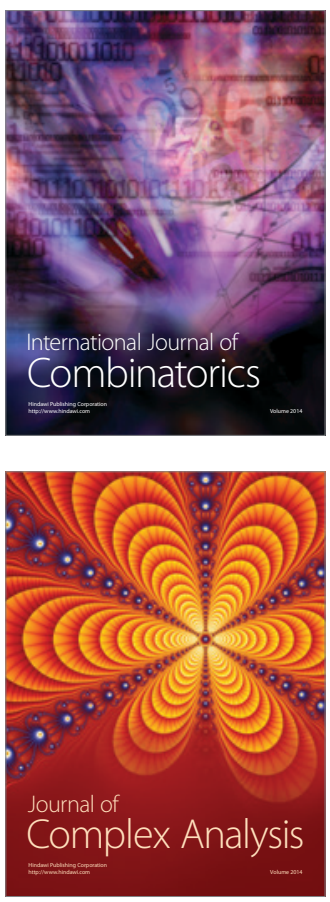

International Journal of

Mathematics and

Mathematical

Sciences
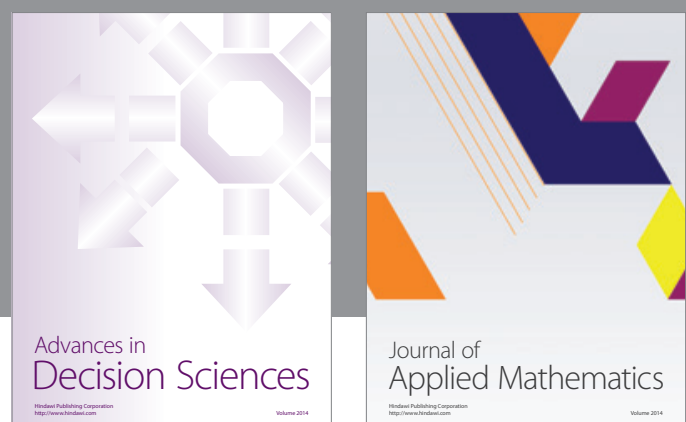

Journal of

Applied Mathematics
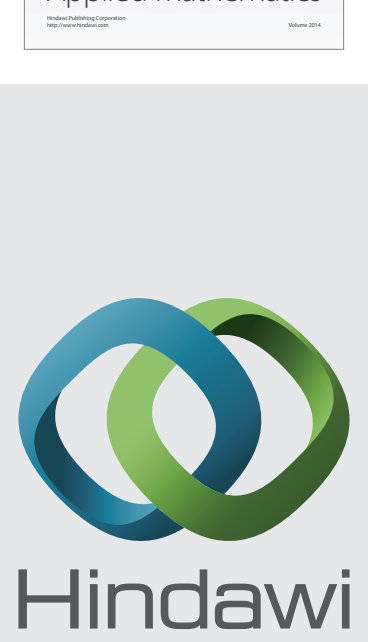

Submit your manuscripts at http://www.hindawi.com
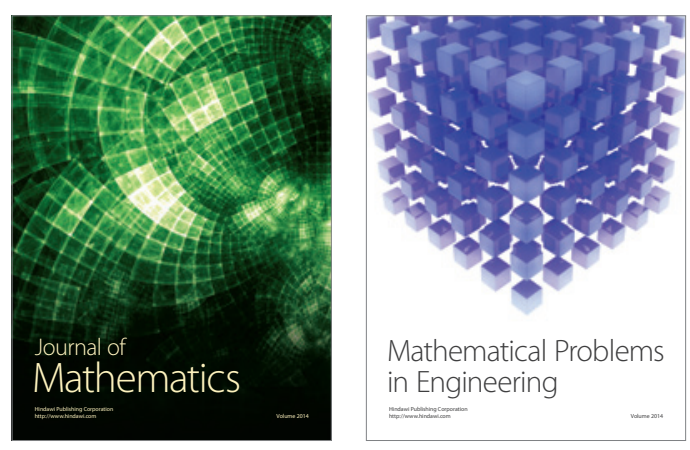

Mathematical Problems in Engineering
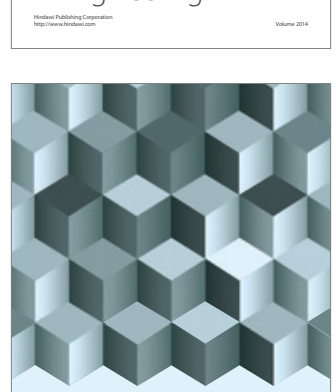

Journal of

Function Spaces
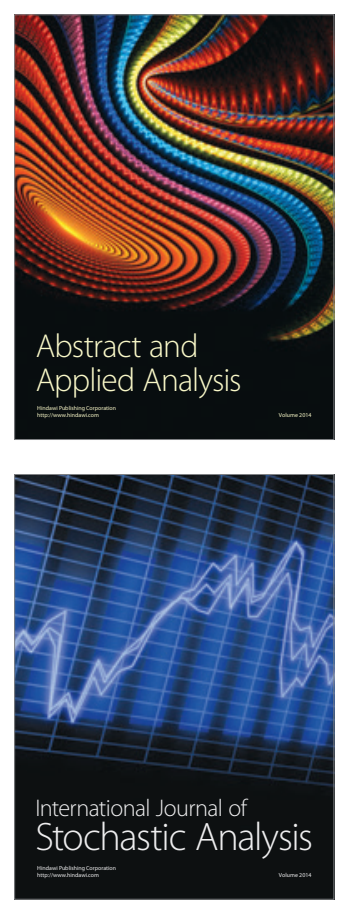

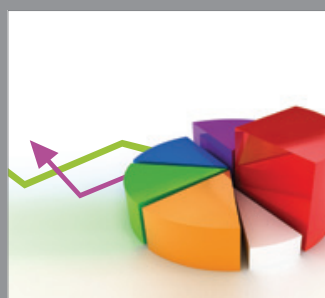

ournal of

Probability and Statistics

Promensencen
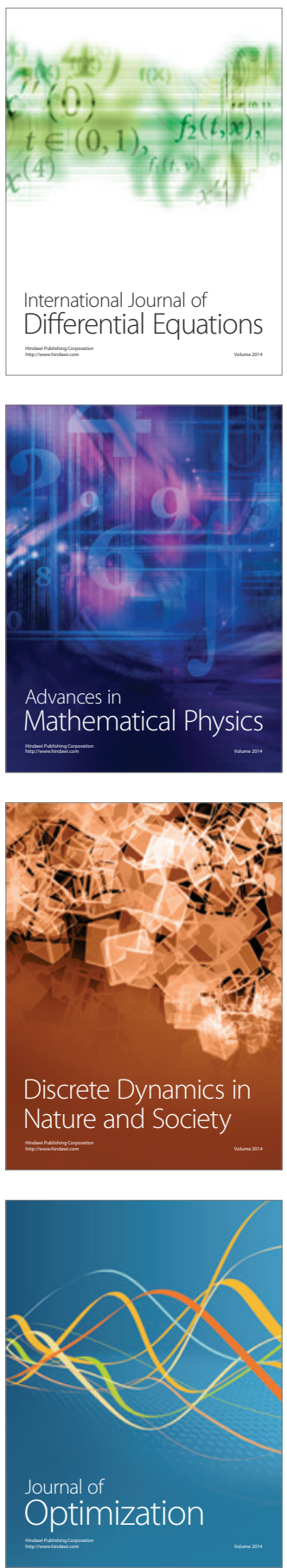